\title{
Editorial
}

\section{The Fragmentation of Integrated Neonatal Intensive Care}

\section{Gilbert I. Martin, MD \\ Jeffrey J. Pomerance, MD, MPH}

\section{Editor's Note:}

Editorials are written today to express views regarding topical issues and to try to elicit comments from the readership. The successful editorial will state an opinion while admitting that there may be other points of view.

In 1983, Jeffrey Pomerance and I wrote the following editorial entitled The Fragmentation of Integrated Neonatal Intensive Care.

Although this was written 16 years ago, I felt the material was important enough to be retrieved from the archives and presented again. I welcome your comments.

GILBERT I. MARTIN, MD

"The extraordinary development of modern science may be her undoing. Specialism, now a necessity, has fragmented the specialties themselves in a way that makes the outlook hazardous. The workers lose all sense of proportion in a maze of minutiae."

—Sir William Osler, address, Classical Association, Oxford, May

16, 1910.

Perinatal morbidity and mortality have decreased rapidly in the past decade. The regionalization of perinatal care into specialized centers has offered medical and emotional benefits to both infants and families but the multidisciplinary approach to perinatology has divided care into many fragments.

Although expertise increases in each particular sector, potential drawbacks may decrease the benefits actually reaped. Inappropriate use of modern technology results when laboratory and monitoring data alone are used in place of patient examination. Additionally, there is all too frequently inadequate communication between subspecialists, which results in parental confusion and professional misinformation.

Citrus Valley Medical Center (G. I. M.), West Covina, CA; and Greater Baltimore Medical Center (J.J. P.), Baltimore, MD.

This article was originally published in Journal of the California Perinatal Association 1983;3:19. Address correspondence and reprint requests to Gilbert I. Martin, MD, Citrus Valley Medical Center, Queen of the Valley Campus, 1115 South Sunset Avenue, West Covina, CA 91793.
In examination of the neonate, emphasis should be placed on the total infant rather than on each separate part. Advanced technology is certainly not error free and diagnosis and management should be based on a "hands-on" approach.

The neonate is now confronted by competing specialists, each focusing on one portion of care, believing that his or her contribution will be additive to the total spectrum. This potentially ideal approach may fail for two reasons. First, the bits of data are difficult to assess and if there is lack of leadership the information will not be distributed efficiently. Second, communication between subspecialists decreases as the number of caretakers increases. Charting is tedious, data dissemination incomplete, and planning difficult. Peripheral disorganization dictates strong central leadership.

Parents today are involved frequently in the day-to-day care of the infant, and the need for bonding, encouragement, and support is emphasized. The parent is faced with multiple problems in addition to the caretaking responsibility. As the number of medical and paramedical personnel increases, conflicting and frequently incorrect or at least incomplete information is provided. Parents listen selectively, and gravitate to the individual who is most accessible and personable and provides hoped-for information. Therefore, a designated communicator should be allocated who is familiar with the total patient and is able to explain to the parents all the aspects concerning diagnosis, therapy, and prognosis. If this does not occur, communication of information becomes fragmented and frequently misleading and incorrect.

Advanced technology is necessary, as is subspecialization. Regular and frequent examination of the neonate is necessary to integrate the many inputs from multiple subspecialties. Close examination of the family is important to successfully deal with the frequent parental aberrations which arise from continual crises facing parents. A wellorganized charting system and regular ongoing multidisciplinary conferences should be established so that the contributions of the team are additive, not fragmented.

The newborn in an intensive care unit is not summation of laboratory data but a human being who should be considered and treated as a whole. Let us not lose sight of the forest for the trees. 